\title{
Identification of threshold concepts involved in early electronics: Some new methods and results ${ }^{*}$
}

\author{
J Scott ${ }^{\dagger}$ and A Harlow \\ University of Waikato, Hamilton, New Zealand
}

\begin{abstract}
This manuscript reports the threshold concepts identified over a two-year study in early circuits and electronics courses. Some novel methods have been used to improve confidence in the identification process. We also identify some concepts, potentially threshold, that ought to have been mastered in high-school physics courses but that are often absent from student repertoires. Weak understanding of these underlying concepts may be a confusing factor for researchers in their search for threshold concepts as well as an additional source of trouble for students of electronics.
\end{abstract}

KEYWORDS: Threshold concepts; electronics; electronics engineering education; modelling; power engineering education; reactive power; ontology.

REFERENCE: Scott, J. \& Harlow, A. 2012, “Identification of threshold concepts involved in early electronics: Some new methods and results", Australasian Journal of Engineering Education, Vol. 18, No. 1.

\section{1}

\section{INTRODUCTION}

To students, threshold concepts (TCs) (Cousin, 2006) are abnormally troublesome to learn. To practitioners their importance lies in the transformation in the "ways of thinking and practising" of the learner as Davies (2006) put it, what we engineers might call acquiring the competencies of our profession. In TC parlance the concepts are said to "be transformative", to possess the power to change the learner's very way of thinking. Meyer (2010) asserted that concepts do not present in a continuum, but fall all-or-nothing into being "threshold" or not. Being both the hardest to learn and the most influential to "identity and ways of thinking" among the ideas within a discipline, it would seem important to both learners and teachers to identify TCs. Once identified, effort can be concentrated on them, and the inordinate learning difficulty anticipated and addressed. The identification proves to be difficult for various reasons (Davies, 2006).

In this manuscript we put forward the small catalogue of concepts we believe to be central to undergraduate

\footnotetext{
* Paper D11-141 submitted 22/12/11; accepted for publication after review and revision 9/05/12.

+ Corresponding author Prof Jonathan Scott can be contacted at scottj@waikato.ac.nz.
}

analogue electronics, and to be threshold. They were identified in the first place by working with students and observing where they reported difficulty, or had learning troubles exposed through assessment. Later each was evaluated against the five attributes of Meyer \& Land (2003). Debate between electrical engineering (EE) educators and instances from the literature have added weight to our selection. Rountree \& Rountree (2009) favoured the search for "the ways in which practitioners in related disciplines solve similar problems" offered by Davies (2006) to identify TCs, and we will go even further than this. This manuscript puts our catalogue of postulated TCs up for debate, presents some novel approaches that we are using to identify TCs, and presents some provocative suggestions regarding irreversibility and integration of TCs.

\section{2}

\section{POSTULATED THRESHOLD CONCEPTS}

We commence by describing the concepts we would offer as TCs, in no particular order, and without attempting to justify the selection. This is to ensure clarity in identification and terminology.

Thévenin's theorem/modelling. Thévenin's theorem is the first example of circuit modelling that students encounter in electronics and circuit theory. It causes 
learners an inordinate amount of trouble. It is not Thévenin: any model would present the difficulty.

Dynamic resistance/linear approximation. The idea that quantities can have an "AC value" as well as a "DC value" appears first and foremost in electronics in the replacement of something like a diode junction with a resistor subject to the limitation of small disturbances; its so-called "dynamic" or small-signal resistance. The same phenomenon - a tangent as a linear approximation of a curve in a local region appears as marginal cost in economics and the $\beta$ of a bipolar junction transistor, and ought to be familiar from differential calculus, but the connection appears seldom to be made.

Reactive power/phasors. Perhaps the most widelyacknowledged TC in EE revolves around the complex nature of impedance and its consequences. The idea of complex electrical quantities is often encountered first by students in the use of phasors. "Reactive power" is a named example that appears commonly in the literature concerning the intellectual effort of applications involving complex quantities. Indeed, it is an idea that continues to cause conceptual difficulty even for experts (Willems, 2011). Like dynamic resistance and the calculus of tangents, this concept ought to come easily on top of complex numbers in mathematics, but the leap is apparently not easy.

Feedback/operational amplification. The operational amplifier (op-amp) is the "simplest" example of feedback. Many practitioners realise that op-amps cause learners a great deal of trouble, but the threshold was there before the op-amp was common. We believe it is the analysis of a circuit with feedback that links the various instances associated with this difficulty.

Transdependence/dependent sources. Dependent sources link $V$ or $I$ of one element with $V$ or $I$ of a separate element. In transistors - "trans-resistors" - it is a transconductance, $g_{m}=\partial i / \partial v$, and students have trouble coming to grips with this. We presume a trans-anything would be equally troublesome.

A further three concepts - potentially threshold - give trouble in early EE courses. We consider these to be strictly not part of EE. They should be encountered and understood in high-school physics courses. In threshold-concept terminology we might say that the students "never completed the liminal passage to full understanding", invoking the idea of a liminal space, postulated to be that state of mind between

1 Simply because the learning of a concept gives trouble to the learner does not necessarily indicate a threshold nature for that concept. If a concept relies upon an earlier idea, lack of understanding of that earlier idea alone can result in trouble learning the new idea. For example, we believe that Kirchoff's laws are not TCs. However, if a student does not already appreciate that charge is conserved, and that free charges in a conductor are not compressible - the holistic view of current flow below - and that current is rate of flow of charge, Kirchoff's laws will be difficult. We consider Kirchoff's laws to be nothing more than tools for attaching quantitative equations to ideas of current flow. encountering a new idea and coming to understand and internalise it. It often falls to the engineering educator to address these ideas as well. ${ }^{1}$

Holistic current flow. We use this name to encompass the physical appreciation that current is charge movement per unit time, that charge is conserved in a conductor, and that, as far as low-frequency electronic circuits are concerned, force of current is conveyed instantly throughout a conductor. The holistic appreciation that current flows "incompressibly" through conductors escapes many students at high school. They seem to think of it as more akin to a gas or to traffic flowing along streets. They can typically recite the rules, but simple circuit questions from a concept exam expose a lack of true understanding. The typical question involves a few light globes wired to a battery. The student may show "localised thinking" and conclude that a change in one part of the circuit will have no effect upon branch currents in a distant ("earlier") part of the circuit (Mazur, 2009). This understanding should not be confused with Kirchoff's rules that simply apply mathematics to obtain quantitative results.

Graph understanding. Some students seem unable to draw even obvious conclusions from graphs, and unable to grasp that a graph and a written sentence are saying the same thing. They may have difficulty reading data off a graph. For example, knowing the load and supply seen by an active component is equivalent to having the load line plotted on the device characteristics, but students have great difficulty transferring between the two representations. As another example, students cannot see why the switching of an ideal diode should yield a plot of a vertical or near-vertical line in one quadrant and horizontal in another quadrant.

$3 D$ to $2 D$ mapping. This skill manifests itself in the EE world in an ability to associate nodes on a circuit diagram with conductors in a constructed circuit. There is a kind of mapping between the physical wires of the three-dimensional construction and the lines on the two-dimensional circuit diagram. This ability resembles that examined in aptitude tests offered to students in the form of questions asking students to say which of several images represents the correct shadow that an object drawn in perspective might cast.

\section{COMPARISON AGAINST ACCEPTED CRITERIA}

The five EE-centric TCs above were initially identified by observing students and listening to their questions and fears - troublesome learning in progress - and comparing notes with other lecturers. We now examine the extent to which each satisfies the four criteria offered by Meyer \& Land (2003) in addition to troublesomeness. 


\subsection{Boundedness}

TCs are said to be bounded, possessing what is described as "terminal frontiers, bordering with thresholds into new conceptual areas", and "defining academic territories" (Meyer \& Land, 2003). Boundedness is quite easily satisfied for most of our candidate concepts. Thévenin's model has a clear boundary: it fails for non-linear or three-terminal circuits. The semiconductor modelling community exists because of these shortcomings. Reactive power, and in particular the representation of it by phasors, has a sharp, clear boundary: calculations only hold for single-frequency, sine-wave excitation. Change the frequency and the answers change and the calculation must be redone. The analysis oversimplifies if harmonics are present. Dynamic resistance fails abruptly to be useful as signals cease to be "small". The very question of what is small can only be answered once you understand the concept. Feedback theory becomes irrelevant the moment the circuit goes unstable. Much of an expert's effort in the field is dedicated to ensuring this very stability, a fact not immediately obvious from the basic theory. The op-amp only became a common component once the burden of guaranteeing stability was assumed by the op-amp designer in the form of "unity-gain compensation", a description that can only be understood once feedback theory is mastered. One can also observe a limitation when stable: The open-loop op-amp transfer function is very hard or impossible to characterise from closedloop measurements. Transdependence has limits that are the least clear amongst our candidate concepts. It has an effective limit in its application: It gives circuit equations that are a jump up in complexity from the case without them, yielding systems that are really only practically solved by computer. ${ }^{2}$

\subsection{Irreversibility}

Irreversibility is hard to assess objectively. Everyone seems to accept the example of riding a bicycle as a skill that cannot be "unlearnt". One senses no degradation in the memory, but there is no guarantee that this is not an illusion. How might we assess this irreversibility? With regard to riding a bicycle, a test would be to find subjects who had not ridden for a long time, and ask them to ride bicycles. ${ }^{3}$

With regard to irreversibility, we chose to interview people who have qualified as engineers but who had long ago ceased to use their skills explicitly. One would expect to find that ideas that cannot be unlearnt will be retained better than other equallyunused ideas. Such subjects are hard to find, and at time of writing our sample space is nine people. Most of the subjects are retired, some had changed discipline from engineering to computing or finance.

We asked subjects about 17 ideas, including our TCs, some concepts taught in EE courses that we are reasonably sure are not threshold, and some ideas from other disciplines. For each concept we established that the subject recognised the idea, typically that they "had once understood it" or believed themselves to have understood it, but that they had not had formal cause to consider or apply it for at least 5 years. ${ }^{4}$ When this was the case we asked them to explain the idea, and assessed whether it "came back" easily or at all. The results have been fascinating. Subjects often claimed to fully understand a concept, but then surprised us and themselves by not being able to describe it to us. Some eventually exposed themselves as having never really understood the idea. To date we have seen no evidence that our TCs are any more irreversible than other concepts.

We suspect that irreversibility may be a derivative property of transformativity. TCs are no more readily remembered than other ideas, but they are frequently used in a subject's routine thinking, so transformed is the subject by the immersion in his or her discipline, that the memory is "dynamically refreshed", in the manner of DRAM. We will say more on this in the section on that attribute below.

\subsection{Integrativeness}

Where an idea recurs in diverse threads, it will necessarily open portals of understanding between the threads, exposing "the previously hidden interrelatedness" in the words of Meyer \& Land (2003). We will substantiate the integrative nature of our postulated TCs by looking at examples of them operating in distant arenas; and especially by identifying them in utterly separate disciplines.

Thévenin's theorem is used as a model for innumerable things from a power station to an electric guitar, from a flashlight battery to an MP3 player. Among EE ideas it is one of the most widely employed of all models. Rossouw et al (2010) found that modelling is one of the most uniformly accepted "unifying concept/themes" in engineering and technology. However, it is easy to provide examples from without engineering. Freud's theory of the mind is

2 Many practitioners may be most familiar with the situation of analysing the operation of a cascade of transistor amplifiers, where we apply Miller's Theorem and other techniques to separate the input-side and output-side parts of the circuit surrounding each transistor and so render the analysis tractable and conceptually insightful (compared to being an emergent property of involved mathematics).

3 We found colleagues who had not ridden for decades, and indeed they were able to mount and ride in a matter of less than 10 seconds, just to check.

4 We are careful to say here "formal cause" to employ the idea. It is entirely possible that irreversibility arises because TCs so transform the practitioner's thinking that they come to use the idea implicitly in everyday life, and thus do not ever have the chance to forget. For example, engineers seem to use the idea of "latent heat of vaporisation" frequently in the kitchen, because their "ways of thinking" have permanently pervaded their culinary habits. 
a (bounded) model of mental processes that brought the concept to psychology. Bohr's orbital model of the atom is another example. Thévenin's theorem, as a model, is easy to establish as an integrating idea.

Dynamic resistance is a term generally limited to the context of active devices in electronics. However, the concept is in wide use by other names in other disciplines. ${ }^{5}$ The dynamic resistance of a device is simply the circuit-theory manifestation of using the tangent of a curve as a local, linear approximation of a non-linear function. It appears to a non-linear control engineer as the response to small disturbances, and to a mechanical engineer a linear approximation of a nonlinear stress-strain relationship. To a radio frequency engineer it appears as S-parameters. To an economist it appears in marginal cost. ${ }^{6}$ In all cases it links into the world of linear approximation of non-linear functions.

Reactive power appears in many unexpected places, indeed it was taken fully-formed from mathematics. Recall that it arises as a way of dealing with differential relationships in the context of periodic excitation: To deal with capacitors and inductors whose voltage and current are related by differential equations the practitioner restricts herself to sinewave excitation and uses complex numbers. In other words, differential equations become linearly soluble in $C$ one frequency at a time. Communications engineers describe the complex modulation that has enabled modern cell-phones and advanced radar with a similar complex mathematics. Invocation of phase in addition to magnitude completes knowledge of reflection coefficients dealing with transmission lines in acoustics and microwave engineering. One can (not so frivolously) reflect that in science fiction the idea has been invoked to explain phenomena that we currently believe impossible: in Star Trek matter disappears when it "goes out of phase", and a device that achieves this was originally called a "phaser".

Feedback occurs everywhere. Before cruise control in cars the driver regulated vehicle speed, a form of

5 Dynamic resistance has other names within electronics. It is sometimes referred to as "AC resistance" or "small-signal resistance".

6 As an aside, we have discovered that use of examples taken from other disciplines can be powerful in helping students realise the difficulty and depth of TCs. A favourite way of helping students to get their heads around dynamic resistance involves asking an equivalent question around marginal cost. We ask the students to work out the cost of a chocolate bar if 10 are bought for $\$ 10$, then if 20 are bought for $\$ 15$. In the latter case the answer will be $75 \mathrm{c}$ instead of $\$ 1$. Then we point out that the first 10 bars cost $\$ 10$, but the second 10 bars cost only an extra $\$ 5$, so surely the last bar cost 50 c not 75 c. Which is right? It depends, of course, but so also does the resistance of something not linear in the relationship of voltage and current.

7 There is an argument that it is simply another instance of modelling, but applied to a three-terminal (or more) network instead of a two-terminal one. We include it separately because there seems to be something freshly confusing to students at its introduction, and we do not accept that it is troublesome purely because the hidden connection must be modelled to be visualised. feedback. An archer improving aim on subsequent shots applies feedback in a sampled system. From horse-riders to pilots, people master the art of steering a system through strange motions, feeding back their perception of their course to the motions to minimise the error between present and desired position and speed. You use feedback to hold your hand still or to eat without spilling. A teacher uses feedback to help students improve their work or self-esteem. The applicability of feedback is enormously widespread, so once mastered it is strongly integrative.

Transdependence is present in the models of all active devices from vacuum tubes to heterojunction bipolar transistors. It is, in essence, remote control, or the apparent connection of two phenomena that could be expected to be disconnected. Viewed this way, it is a wormhole or the Klein bottle to a topologist, the prestige of a trick to a magician, or perhaps even akin to indirection to a computer scientist. The concept has a universal applicability. ${ }^{7}$

\subsection{Transformativity}

Various methods have been applied to gauge the transformativity of concepts. Davies \& Mangan (2005) looked at concepts historically featured in exams. They also looked for naïveté in qualitative student responses compared with expert's responses around candidate concepts, associating gradual liminal passage away from naïveté with the transformation. Atherton et al (2008) suggested that practitioners of "hard disciplines" such as engineering identify TCs through introspection with greater ease and consistency than softer disciplines. Davies (2006) concentrated on the transformative aspect of TCs. He seemed sceptical of taking a consensus approach, even with expert teachers, but in the end he offered mostly introspective solutions. How can we say that an understanding of reactive power (or some other candidate concept) has changed another person's way of thinking? Introspection is the main option simply because few of us has close enough a view of another individual throughout the transformation. Kiley (2009) offered perhaps the best opportunity to identify TCs through the relationship between doctoral candidates and their supervisors that lasts 3-5 years and involves a close working situation. Most researchers settle for the view over a single course, typically a semester.

The literature portrays transformative discipline ideas as those that give rise to characteristic ways of thinking. We suggest that if this is the case, they will be used by practitioners in disparate situations, because to be transformative they must be powerful. Out-of-context applications can pinpoint deeply-influential concepts. For example, experts tend to betray their professions in "dinner party conversations" through their use of their "ways of thinking" and listeners can identify new acquaintances across a dinner table as psychiatrists, 
economists, engineers, or whatever, simply from the way they respond to provocative problems by applying their characteristic "ways of thinking", or concepts and associated specialist language stereotypical of their community of expertise. Part of the appeal of entertainment programs such as "The Big Bang Theory" is the appearance of professionrelated mental approaches out of context in social situations. Sadly, it would take a long time to identify TCs using these methods, but we can provide examples where the essence of our TCs appears in other disciplines or in stereotypical behaviours. (Space does not permit our full set of examples.)

In the case of Thévenin/modelling, we have cited "foreign" examples such as Freud's models of the mind or Bohr's atom. These transformed their entire disciplines. Rossouw et al (2010) would have modelling taught as a mental skill or attribute to educate technologists, in effect building in the way of thinking first and applying it later. At the other end of the scale, modelling is used to explain hygiene to children with germs as small creatures, or in a simplified block diagram of the components of a home theatre system. These are ways of thinking about or explaining phenomena of everyday life.

Regarding dynamic resistance, there is a pervasive desire to use linear approximation: virtually all disciplines have their "linear approximations". The water requirements of plants are a subtle function of sunlight, temperature, wind and humidity, but if a gardener is asked, he will say that $20 \%$ more sunlight might demand $20 \%$ more water. It is a way of thinking, a common line of intellectual attack on any problem. There is a pull to use it even where instruction indicates that it is both wrong and risky: if one dose of medicine improves a condition, the implication is that double the dose will double the benefit.

Feedback is tacitly employed widely. So transformed is the professional's mindset that there seems to be no part of daily life where the lessons of feedback are forgotten. When the engineer uses a new toaster, she starts with the control low, and adjusts based on the colour of the toast "samples". The doctor might see the shake in a person's out-held hand as loop instability, and the engineer sees caffeine as increasing gain and likely to worsen the shake. Anyone might tell you that you are more likely to spill a cup of water that you are carrying if you look directly at it, but the engineer adds to the conversation "because your response time to perceived movement of the liquid is of the same order as the natural time constant of the water in the cup", and many an engineering listener will not think the comment unusual.

Reactive power, or at least the appreciation of phase, is as pervasive as feedback. The idea can be used as a communication shortcut. For example, suffering from jet lag and wanting to wake up at night and go to sleep in the day is succinctly described as having your "body clock out of phase with your wristwatch".
Transdependence is more difficult with respect to transformativity. Consider that hygiene or sterility is suggested as a TC in nursing (Atherton et al, 2008). This seems ludicrous at first, for most of us have had the idea of something being "dirty" and not to be eaten or exposed to our bodies once it has touched the ground, from a very young age. This was not always the case, and remains not the case in some cultures. Could it be that the underlying remote-control action embodied by transconductance, once amazing, is taken for granted in this age of infrared cordless and wirelessly-connected world? Once people were amazed that the flicking of a switch could conjure light, yet today we barely react when pictures appear on our iPads through the aether. We are transformed in our thinking at so young an age, and so universally, that the transformation goes unremarked. To see the transformation wrought by an understanding and acceptance of transdependence requires comparing people's thinking now with the thinking of a century or more ago.

\section{QUANTITATIVE IDENTIFICATION}

We have started investigating two potential quantitative indicators. The first is the doublepeaked characteristic that one often sees in the grade distribution. Scott et al (2010) showed results that suggested that a bimodal grade distribution will appear in assessment involving a single TC. A largerscale study is planned for 2012.

The second indicator involves topological metrics applied to concept maps constructed around the discipline. Concept maps are a structured way of representing a series of statements about ideas in a discipline in the form of a network graph (Novak \& Gowin, 1984). Two concepts (nodes) are linked through an action or dependency. For example, "dynamic resistance depends upon operating point" would create a link between the concepts of dynamic resistance and operating point. The analysis of networks has become an area of intense interest in the last decade or so, with many characterisation metrics available that can be readily applied to a connected network graph (Cui et al, 2010). We suggest that the degree (of connectedness) of nodes associated with TCs will differ from that of non-threshold ones. The reasoning is that the map will evidence the integrative property of the TCs as an increased number of edges or links to those particular nodes. The analysis could resemble the assessments of connectedness after the fashion of the well-known Erdös and Bacon numbers familiar to mathematicians and film buffs. The problem is that topological metrics are meaningful only when applied to large networks. Constructing a concept map that is large enough for the metrics to reflect the integrative nature of some concepts is proving to be a challenge. Some initial work has been reported by Jaffer et al (2012). 


\section{5}

CORRELATION WITH EXISTING CONCEPT CATALOGUES

The idea of a "concept inventory" was introduced 20 years ago by Hestenes et al (1991), in the area of physics, with the Force Concept Inventory. A number of such inventories have appeared in disciplines ranging from thermodynamics to chemistry (Evans et al, 2003). These inventories typically lead to an assessment tool designed to assess understanding; in TC terminology one might say to assess that the user has passed through the portal with respect to each of a series of concepts considered important to the discipline. The Foundation Coalition project has been associated with a number of the tools (Corleto et al, 1996). Some are developed and maintained more effectively than others. For example, the Signals and Systems Concept Inventory, associated with a 25-question tool, is well researched and identifies the concepts it tests very explicitly (Wage et al, 2005)

Simoni et al (2004) reported on an Electronics Concept Inventory. In an attempt to see if our ideas of TCs correlate with the concepts considered important by the contributors to the index, we obtained the exam of Simoni et al (2004) and looked for the appearance of our concepts. The Electronics Concept Inventory does not address reactive power (it is usually considered to be "circuit theory" rather than electronics), but it does use Thévenin's theorem and the equivalence of Thévenin's equivalent circuit as a model, the transaction of dependent sources, the action of op-amp circuits. It implicitly assumes a knowledge of dynamic resistance, and of its change with operating point of a non-linear element. It also implicitly requires an ability to discern the action of a circuit from graphs. We consider the Electronics Concept Inventory to endorse the importance of four of our five concepts, while eschewing comment upon the fifth.

We looked into the Electric Circuits Inventory of Ogunfunmi \& Rahman (2010). This inventory was limited to DC circuits, and so did not extend to reactive power and could shed no light.

\section{SUMMARY AND CONCLUSIONS}

If the threshold nature of certain concepts is to be used to refine or revolutionise curricula, we need to identify the concepts. This paper proposes that there are five TCs in early analogue electronics. We identify these concepts. In the justification of this selection we present some new approaches and thinking. We suggest that irreversibility may be no more than a derivative of a basic attribute, rather than a basic attribute in its own right (as perhaps is discursivity). We also observe that our postulated TCs can be discerned in other disciplines, often far removed from engineering. This raises the possibility that TCs may be so integrative because they run across disciplines.

\section{REFERENCES}

Atherton, J., Hadfield, P. \& Meyer, R. 2008, “Threshold Concepts in the Wild", Threshold Concepts: from Theory to Practice Conference, Queen's University, Kingston, Ontario, 18-20 June, www.doceo. co.uk/tools/ Threshold_Concepts_Wild_expanded_70.pdf.

Corleto, C. R., Kimball, J. L., Tipton, A. R. \& MacLauchlan, R. A. 1996, "Foundation Coalition First Year Integrated Engineering Curriculum at Texas A\&M University-Kingsville: Development, Implementation and Assessment", Frontiers in Education 26 $6^{\text {th }}$ Annual Conference, 6-9 November, Vol. 3, pp. 1141-1145.

Cousin, G. 2006, "An introduction to Threshold Concepts", Planet, No. 17, December, www.gees. ac.uk/planet/p17/gc.pdf.

Cui, L., Kumara, S. \& Albert, R. 2010, “Complex Networks: An Engineering View", IEEE Circuits and Systems Magazine, No. 3.

Davies, P. 2006, "Threshold Concepts: How can we recognise them?", Overcoming barriers to student understanding: Threshold concepts and troublesome knowledge, Meyer, J. \& Land, R. (editors), Routledge, London.

Davies, P. \& Mangan, J. 2005, “Recognising Threshold Concepts: an exploration of different approaches", presented at the European Association in Learning and Instruction Conference (EARLI), 23 August, Nicosia, Cyprus.

Evans, D. L., Gray, G. L., Krause, S., Martin, J., Midkiff, C., Notaros, B. M., Pavelich, M., Rancour, D., Reed-Rhoads, T., Steif, P., Streveler, R. \& Wage, K. 2003, "Progress on concept inventory assessment tools", Frontiers in Education, 5-8 November, Vol. 1, No. T4G, pp. 1-8.

Hestenes, D., Wells, M. \& Swackhamer, G. 1991, "Force Concept Inventory", The Physics Teacher, Vol. 30, No. 3, pp. 141-151.

Jaffer, Z., Fill, J., Male, S., Quinlan, K., Stamboulis, A., Davis, C. \& Baillie, C. 2012, "Discovering the thresholds in materials education", $4^{\text {th }}$ International Symposium in Materials Engineering Education, Cambridge, UK, 12-13 April.

Kiley, M. 2009, "Identifying threshold concepts and proposing strategies to support doctoral candidates", Innovations in Education and Teaching International, Vol. 46, No. 3, pp. 293-304.

Mazur, E. 2009, "Confessions of a Converted Lecturer", presented at Waterloo University, www. youtube.com/watch?v=WwslBPj8GgI, accessed February 2012. 
Meyer, J. 2010, personal communication.

Meyer, J. \& Land, R. 2003, “Threshold Concepts and Troublesome Knowledge: linkages to ways of thinking and practicing within the disciplines", Improved Student Learning - Ten Years On, Rust, C. (editor), OCSLD, Oxford.

Novak, J. D. \& Gowin, D. B. (editors), 1984, “Concept mapping for meaningful learning", Learning how to learn, Cambridge University, New York, pp. 15-54.

Ogunfunmi, T. \& Rahman, M. 2010, “A Concept Inventory for an Electric Circuits Course: Rationale and Fundamental Topics", Proceedings of the IEEE International Symposium on Circuits And Systems (ISCAS), 30 May to 2 June 2, Paris, pp. 2804-2807.

Rossouw, A., Hacker, M. \& de Vries, M. J. 2010, "Concepts and contexts in engineering and technology education: an international and interdisciplinary Delphi study", International Journal of Technology and Design Education, Springer, Netherlands, July, doi:10.1007/s10798-010-9129-1.
Rountree, J. \& Rountree, N. 2009, “Issues Regarding Threshold Concepts in Computer Science", Proc. Eleventh Australasian Computing Education Conference (ACE 2009), Wellington, New Zealand, Hamilton, M. \& Clear, T. (editors), pp. 139-145.

Scott, J., Harlow, A., Peter, M. \& Cowie, B. 2010, "Threshold Concepts and Introductory Electronics", Proceedings of the 21 $1^{\text {st }}$ AaeE Conference, 5-8 December, Sydney.

Simoni, M. F., Herniter, M. E. \& Ferguson, B. A. 2004, "Concepts to Questions: Creating an Electronics Concept Inventory Exam", Proceedings of the 2004 American Society for Engineering Education Annual Conference \& Exposition, Session 1793.

Wage, K. E., Buck, J. R., Wright, C. H. G. \& Welch, T. B. 2005, "The Signals and Systems Concept Inventory", IEEE Transactions on Education, Vol. 48, No. 3, pp. 448-461.

Willems, J. L. 2011, “Budeaunu's Reactive Power and Related Concepts Revisited", IEEE Trans. on Instrumentation and Measurement, Vol. 60, No. 4, April, pp. 1182-1186. 


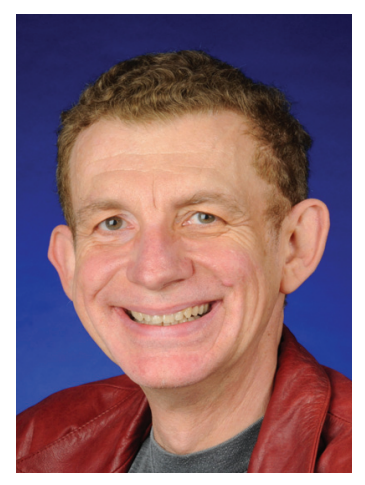

\section{JONATHAN SCOTT}

Prof Jonathan Scott became the Foundation Professor in Electronic Engineering at the University of Waikato in Hamilton, New Zealand, in 2006. Prior to this he was with Hewlett-Packard and Agilent Technologies in California. His interests include metrology, compound semiconductor integrated circuit design, embedded systems, microwaves, engineering education, power harvesting and mechatronics. He has served on committees of the Standards Association of Australia, and the NRC Review Panel for the National Institute of Standards and Technology. He is a Professorial Fellow of Macquarie University. He received a faculty teaching excellence award in 2011 from the University of Waikato. Jonathan teaches a number of undergraduate courses in electronics, supervises several graduate students, has authored over 100 refereed publications, and he holds a number of patents.

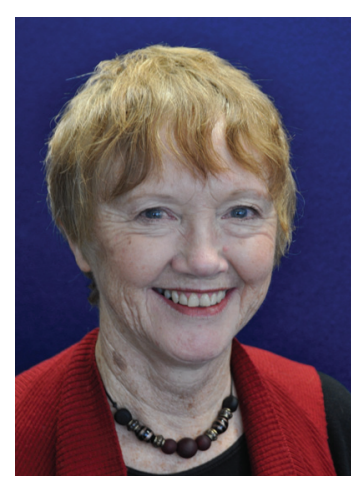

\section{ANN HARLOW}

Since 2001, Ann Harlow has been a Senior Research Officer at the Wilf Malcolm Institute of Educational Research in the School of Education at the University of Waikato, where she specialises in educational research design and implementation. She has a particular interest in teaching and learning at tertiary level. Ann has worked on more than 15 major research project teams for the University of Waikato, covering a range of educational topics related to curriculum, learning and teaching, and assessment. For the last two years, Ann has been the principal investigator on the threshold concepts research project funded by the Teaching and Learning Research Initiative, with responsibilities for designing the research in collaboration with tertiary teachers. 